\title{
The Effect of High Flux versus Low Flux Dialyzers on Serum Fibroblast Growth Factor-23 (FGF-23) and Its Cardiovascular Implications in Prevalent Hemodialysis Patients \\ Walid Ahmed Bichari, Mariam Gamal Aabed, Ashraf Hassan Abdelmobdy* \\ Department of Internal Medicine, Faculty of Medicine, Ain Shams University \\ *Corresponding author: Ashraf Hassan Abdelmobdy, Mobile: (+20)01093989048, E-Mail: ashrafnephro@yahoo.com
}

\begin{abstract}
Background: Cardiovascular disease is the main cause of death in patients with end stage renal disease. Fibroblastic growth factor- 23 (FGF-23) is a novel phosphaturic hormone produced in the osteocyte. Patients with kidney disease complain of elevated levels of FGF-23 in response to hyperphosphatemia.

Patients and Methods: This was a cross-over prospective study conducted on 30 patients on regular hemodialysis. The study period was 6 months, laboratory investigations for FGF-23, complete blood picture, s. urea (pre-dialysis and post-dialysis), serum albumin, serum electrolytes $\left(\mathrm{Ca}^{++} \& \mathrm{PO}_{4}\right)$, alkaline phosphatase, $\mathrm{PTH}$, serum ferritin, and lipid profile as well as echocardiography were obtained in the start of the study, after 3 months on maintained lowflux hemodialysis and 3 months after switching the patients to high-flux hemodialysis.

Results: There was a highly significant reduction of FGF-23 level with high-flux dialyzers than with low-flux dialyzers. The adjusted difference in the absolute change in FGF-23 levels between the three months period of lowflux dialysis and the three months period of high-flux dialysis showed a highly significant reduction with high-flux than with low-flux $(\mathrm{p}=0.000)$. There was a significant reduction of serum levels of PTH, calcium, phosphorus levels with high-flux dialyzers than with low-flux dialyzers, the adjusted difference was $(\mathrm{p}=0.022),(\mathrm{p}=0.000)$, and $(\mathrm{p}=$ 0.006) respectively.

Conclusion: FGF-23 levels were significantly higher in hemodialysis patients than in normal population. FGF-23 was better eliminated by high-flux dialyzers than low-flux dialyzers and high FGF-23 levels were associated with increased incidence of cardiac valve calcification. FGF-23 positively correlated with right ventricular systolic pressure.
\end{abstract}

Keywords: Cardiovascular, Low-flux, High-flux, FGF-23, Hemodialysis patients

\section{INTRODUCTION}

Patients with end stage renal disease, treated with hemodialysis, have an annual mortality rate of approximately 20\%. Cardiovascular disease is the main cause of death, with the annual death rate from CVD at 9\%, which is approximately 10-20 higher than in the general population ${ }^{(1)}$. Vascular calcification is also common in patients with end-stage renal disease (ESRD) who are treated with regular dialysis and it may contribute to the very high mortality rate from cardiovascular causes in such patients. Arterial calcification can result from the deposition of mineral along the intimal layer of the arteries in conjunction with atherosclerotic plaques or from calcium deposition in the medial layer of the arteries that is due, at least in part, to disturbances in mineral metabolism (2).

fibroblastic growth factor 23 (FGF23) is a novel phosphaturic hormone produced in the osteocyte $^{(3)}$. In patients with kidney disease, normal serum phosphate levels are maintained despite a declining nephron mass, in part by progressive "secondary" increases in FGF-23 levels ${ }^{(4)}$. Increases in FGF23 levels help maintain serum phosphate in the normal range in CKD. Prospective studies in

Received:11/1/2020

Accepted:20/3/2020 populations of pre-dialysis $\mathrm{CKD}$, incident and prevalent ESRD and kidney transplant recipients demonstrate that elevated FGF23 levels are independently associated with progression of CKD and development of cardiovascular events and mortality ${ }^{(5)}$.

Aim of study to compare between the effect of low-flux versus high-flux dialyzers on serum level of FGF-23, and to study the association between FGF-23 and echocardiographic parameters in hemodialysis patients.

\section{PATIENTS AND METHODS}

This was a cross-over prospective study conducted on 30 patients on regular hemodialysis maintained on thrice weekly sessions in Dar Elshefaa hemodialysis unit, 4 hours each. The study Period was 6 months (from March to August 2016). Patients were on low flux polysulfone filters (Fresinius F6), then switched to high-flux polysulfone filters (F6). Laboratory investigations included FGF-23, complete blood picture, s. urea (pre-dialysis and post-dialysis), serum albumin, serum electrolytes $\left(\mathrm{Ca}^{++} \& \mathrm{PO}_{4}\right)$, alkaline phosphatase, PTH, serum ferritin, and lipid 
profile as well as echocardiography were obtained in the start of the study, after 3 months on maintained low-flux hemodialysis and 3 months after switching the patients to high-flux hemodialysis.

Inclusion criteria: The patients should be $\geq 18$ years old and maintained on regular HD for more than 6 months.

Exclusion criteria: Active infection, active autoimmune disease, malignancy, decompensated liver disease, and heart failure class III and IV.

All patients were subjected to full history taking including medical co-morbidities, etiology of renal failure, regimen of hemodialysis, duration on dialysis and complications related to hemodialysis.

\section{Ethical consideration and written informed consent:}

An approval of the study was obtained from Ain Shams University academic and ethical committee. Every patient signed an informed written consent for acceptance of the operation.

\section{Statistical analysis}

Recorded data were analyzed using the statistical package for social sciences, version 20.0 (SPSS Inc., Chicago, Illinois, USA). Quantitative data were expressed as mean \pm standard deviation (SD). Qualitative data were expressed as frequency and percentage.

\section{The following tests were done:}

- Independent-samples t-test of significance was used when comparing between two means.

- Chi-square $\left(\mathrm{x}^{2}\right)$ test of significance was used in order to compare proportions between two qualitative parameters.

- The confidence interval was set to $95 \%$ and the margin of error accepted was set to $5 \%$. The p-value was considered significant as the following:

- Probability (P-value)

- P-value <0.05 was considered significant.

- P-value <0.001 was considered as highly significant.

- P-value >0.05 was considered insignificant.

\section{RESULTS}

Among the included 30 patients, there was 16 male patients $(53.3 \%)$ and 14 female patients $(46.7 \%)$ and the mean hemodialysis duration was $5.22 \pm 3.39$ years with hemodialysis duration ranging from 1 year to 13 years. Etiology of ESRD was hypertension (HTN) 18 patients $(60.0 \%)$, diabetes mellitus (DM) 4 patients (13.3\%), polycystic kidney disease (PKD) 3 patients (10.0\%), glomerulonephritis (GN) 2 patients (6.7\%), obstructive uropathy 1 patient $(3.3 \%)$ and unknown cause was in 2 patients $(6.7 \%)$. The baseline FGF-23 levels were higher than the normal range (Median 400 \& IQR: 266.5 - 531)

There was a highly significant reduction of FGF-23 level with high-flux dialyzers than with lowflux dialyzers. The difference in the absolute change in FGF-23 levels between the three months period of low-flux dialysis and the three months period of highflux dialysis showed a highly significant reduction with high-flux than low-flux ( $\mathrm{p}=0.000)$ as shown in table (2) and figure (6).

There was a significant reduction of serum levels of PTH, calcium and phosphorus levels with high-flux dialyzers than with low-flux dialyzers. The adjusted difference was $(\mathrm{p}=0.022),(\mathrm{p}=0.000)$, and $(\mathrm{p}=0.006)$ respectively (Table $2 \&$ figures $3,4 \& 5)$.

Also there was significant increase in serum albumin, and significant reduction in total cholesterol level with high flux dialyzers than with low flux dialyzers. When we compared the adjusted difference of serum albumin level during the low-flux period and the high-flux period, (median -0.1, IQR $(-0.2-0.1)$ ) and (median 0.05, IQR $(-0.1-0.3)$ ) respectively, it showed a significant increase in serum albumin when using high-flux compared to low-flux dialyzer, $(\mathrm{p}=$ 0.049) (Table $1 \&$ figure 1 ).

As regards lipid profile, when using the adjusted difference between the low-flux and high-flux period there was a significant reduction of the total cholesterol during the high-flux period ( $\mathrm{p}=0.038)$, [median 8 (IQR -5 - 31)] and [median 4 (IQR -1013)] respectively (Table $1 \&$ figures 2 ).

As regards echocardiography findings, with the use of high-flux dialyzer, there was a decrease in the number of patient with diastolic dysfunction although it did not reach a significant value. The adjusted difference between low-flux and high-flux periods showed a significance improvement in ejection fraction value $(\mathrm{p}=0.031)$ (Table 3$)$.

FGF-23 showed a positive correlation with RVSP at baseline, after 3 months on low-flux dialysis, and after 3 months on high-flux dialysis with P-value (0.000), (0.001), and (0.000) respectively (Figure 8).

In our study, we found a highly significant correlation between FGF23 level and valvular calcification with low-flux dialyzer. Also, there was a significant correlation between FGF23 level and valvular calcification with the high-flux dialyzer (Table 3 and figures $7 \& 8$ ).

In our study. FGF-23 showed a negative correlation with baseline total cholesterol and LDL levels $(\mathrm{p}=0.048)$ and $(\mathrm{p}=0.036)$ respectively (Table 5 and figures 10 and 11). 
Table (1): Comparison between the difference in laboratory results of the patients during the period of low-flux dialysis and the difference during the high-flux dialysis.

\begin{tabular}{|c|c|c|c|c|c|c|}
\hline \multicolumn{2}{|c|}{} & $\begin{array}{c}\text { Diff. between } \\
\mathbf{0 - 3}\end{array}$ & $\begin{array}{c}\text { Diff. between } \\
\mathbf{3 - 6}\end{array}$ & \multirow{2}{*}{$\begin{array}{c}\text { Test value } \\
\neq\end{array}$} & $\begin{array}{c}\text { P- } \\
\text { value }\end{array}$ & Sig. \\
\cline { 3 - 6 } & (low-flux) & (high-flux) & & \\
\hline HGB (gm/dl) & Median (IQR) & $-0.2(-1-0.7)$ & $0.05(-1.1-0.6)$ & -0.141 & 0.888 & NS \\
\hline Albumin (mg/dl) & Median (IQR) & $-0.1(-0.2-0.1)$ & $0.05(-0.1-0.3)$ & -1.972 & 0.049 & S \\
\hline Ferittin (ng/ml) & Median (IQR) & $-15(-176-40)$ & $-10(-120-100)$ & -0.991 & 0.322 & NS \\
\hline Total Cholesterol (mg/dl) & Median (IQR) & $8(-5-31)$ & $4(-10-13)$ & -2.07 & 0.038 & S \\
\hline Triglyceride (mg/dl) & Median (IQR) & $-2(-27-16)$ & $-19.5(-37-12)$ & -1.198 & 0.231 & NS \\
\hline LDL (mg/dl) & Median (IQR) & $1.8(-4.8-22.6)$ & $6.4(-6-14.4)$ & -0.488 & 0.626 & NS \\
\hline HDL (mg/dl) & Median (IQR) & $-0.5(-3-4)$ & $3.5(0-8)$ & -1.912 & 0.056 & NS \\
\hline
\end{tabular}

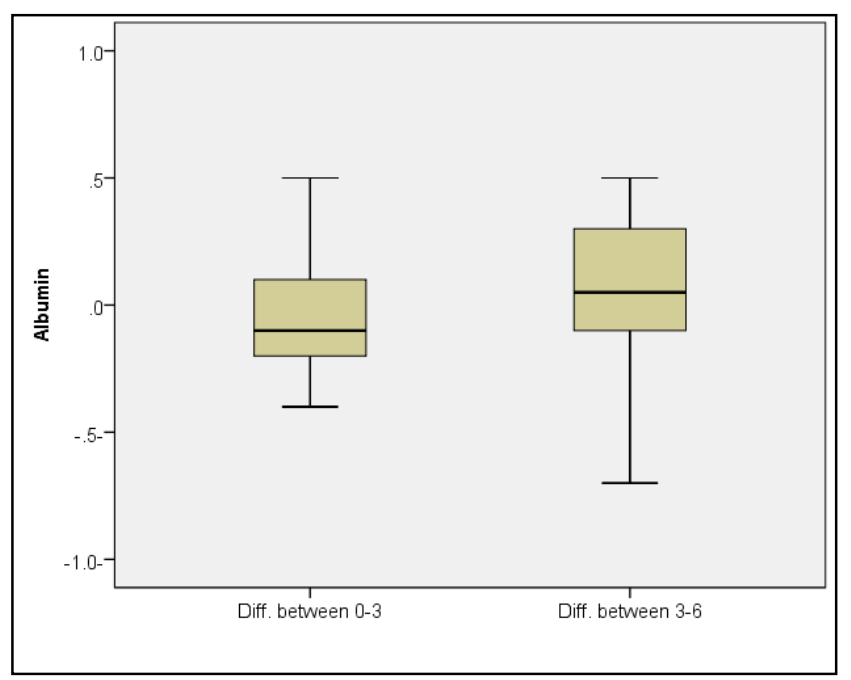

Figure (1): Comparison between the difference in serum albumin level during the period of low-flux dialysis and the difference during the high-flux dialysis.

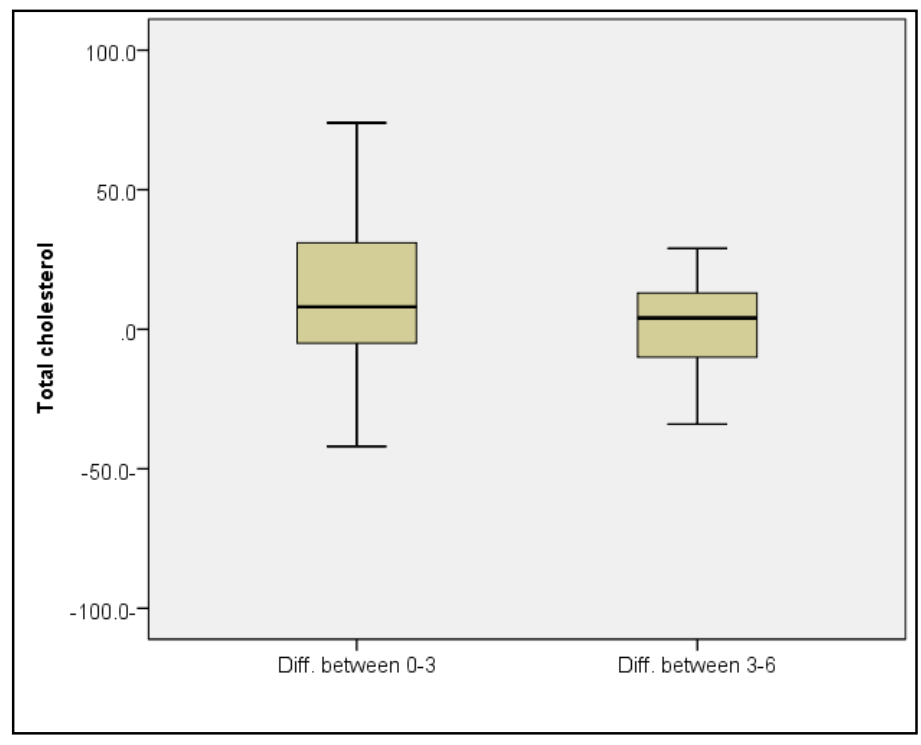

Figure (2): Comparison between the difference in total cholesterol level during the period of low-flux dialysis and the difference during the high-flux dialysis. 
Table (2): Comparison between the differences in laboratory results of the patients during the period of low-flux dialysis and the difference during the high-flux dialysis as regard the specific biomarkers of mineral and bone metabolism.

\begin{tabular}{|c|c|c|c|c|c|c|}
\hline & \multirow{2}{*}{\begin{tabular}{|c|} 
Diff. between 0-3 \\
(low-flux) \\
\end{tabular}} & \multirow{2}{*}{$\begin{array}{c}\text { Diff. between 3-6 } \\
\text { (high-flux) }\end{array}$} & \multirow{2}{*}{$\begin{array}{c}\text { Test } \\
\text { value } \neq\end{array}$} & \multirow{2}{*}{ P-value } & \multirow{2}{*}{ Sig. } \\
\hline & & & & & & \\
\hline Intact PTH $(\mathrm{pg} / \mathrm{ml})$ & Median (IQR) & $-16.65(-36-5.3)$ & $-49(-92--13.70)$ & -2.284 & 0.022 & $\mathrm{~S}$ \\
\hline Corrected Ca (mg/dl) & Median (IQR) & $0.5(0.3-0.8)$ & $-0.1(-0.3-0.4)$ & -4.135 & 0 & HS \\
\hline $\mathrm{PO}_{4}(\mathrm{mg} / \mathrm{dl})$ & Median (IQR) & $0.45(-0.6-1.2)$ & $-0.5(-1.6-0.2)$ & -2.745 & 0.006 & $\mathrm{HS}$ \\
\hline$A L K . P h(U / L)$ & Median (IQR) & $-4(-37-17)$ & $-28.5(-50-10)$ & -1.871 & 0.061 & NS \\
\hline$F G F-23(p g / m l)$ & Median (IQR) & $-14(-45-0)$ & $-105.3(-205.1--59)$ & -3.692 & 0.001 & HS \\
\hline
\end{tabular}

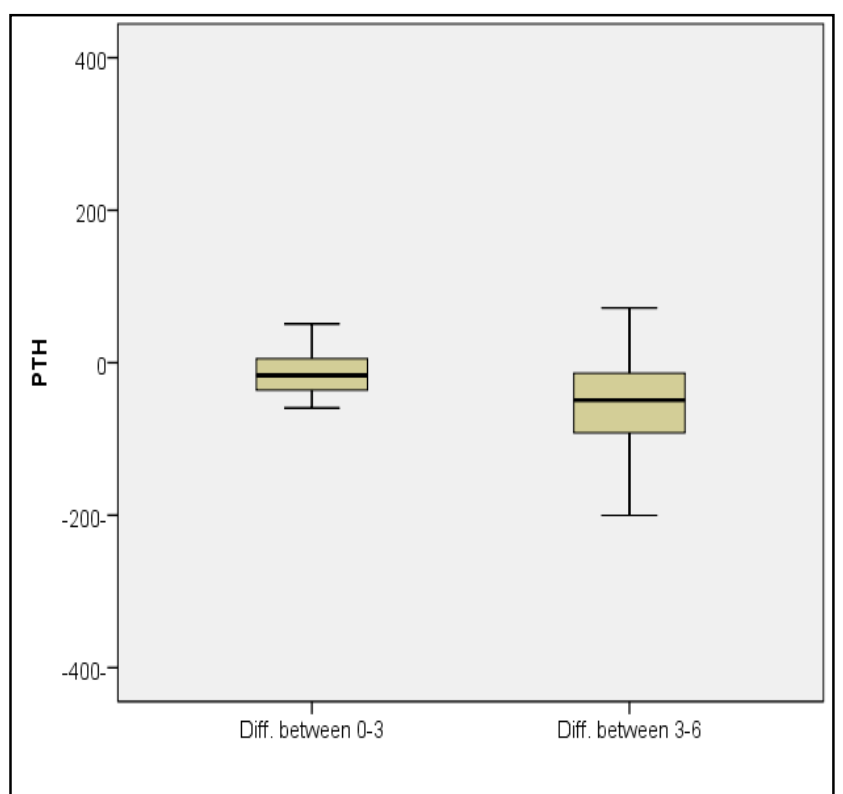

Figure (3): Comparison between the difference in PTH level during the period of low-flux dialysis and the difference during the high-flux dialysis

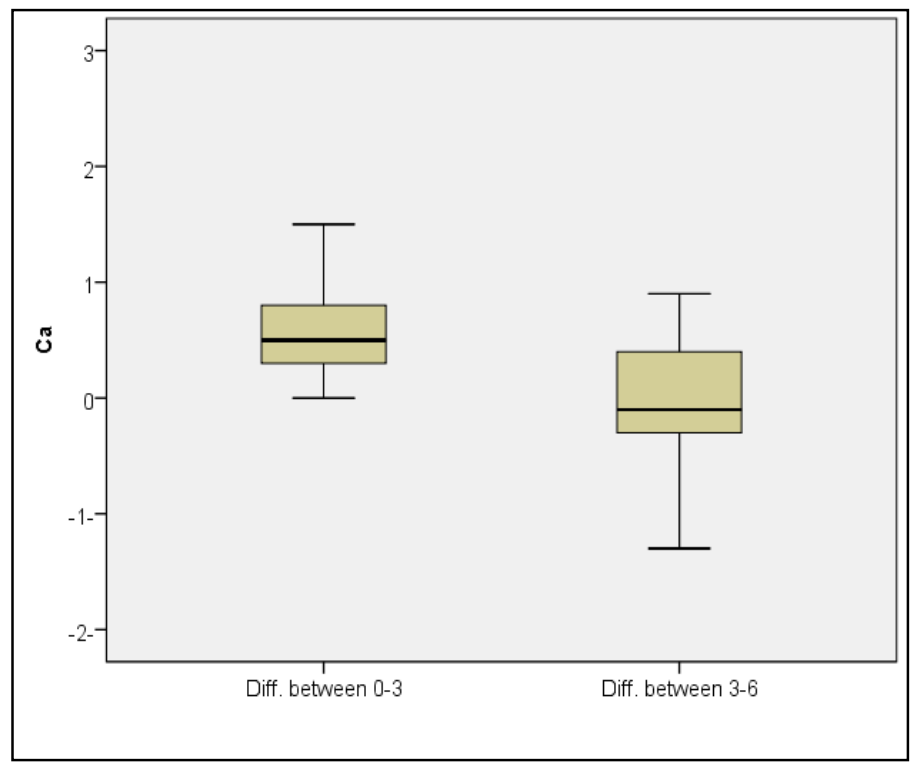

Figure (4): Comparison between the difference in serum calcium level during the period of low-flux dialysis and the difference during the high-flux dialysis. 


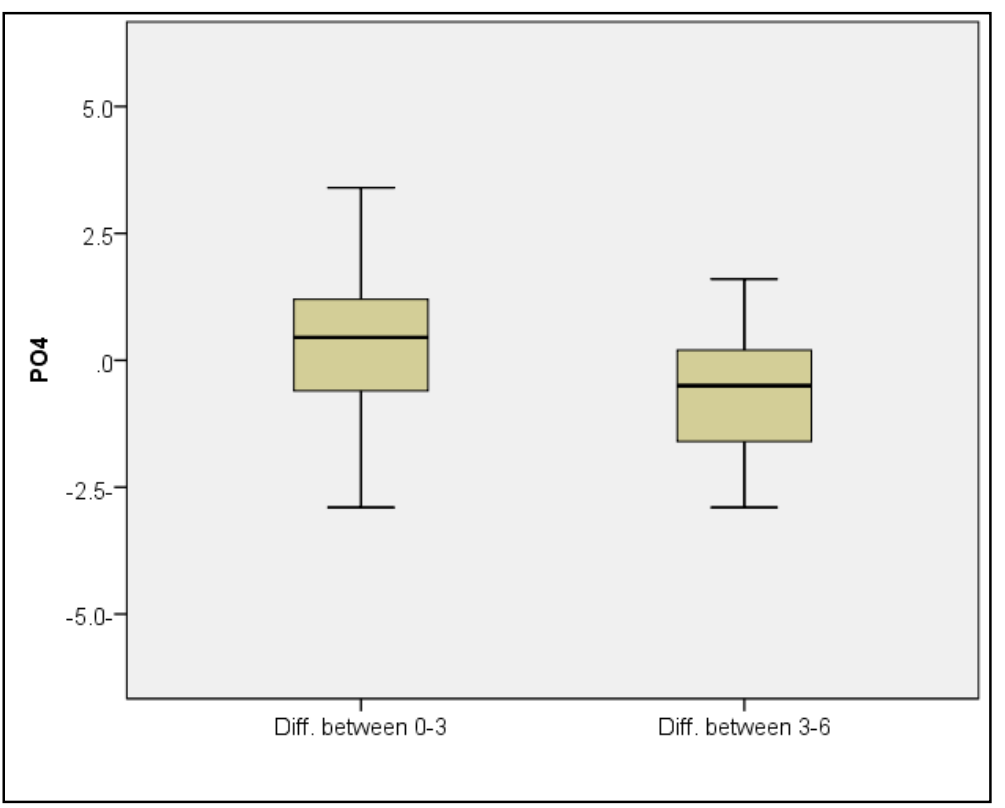

Figure (5): Comparison between the difference in serum phosphorus level during the period of low-flux dialysis and the difference during the high-flux dialysis

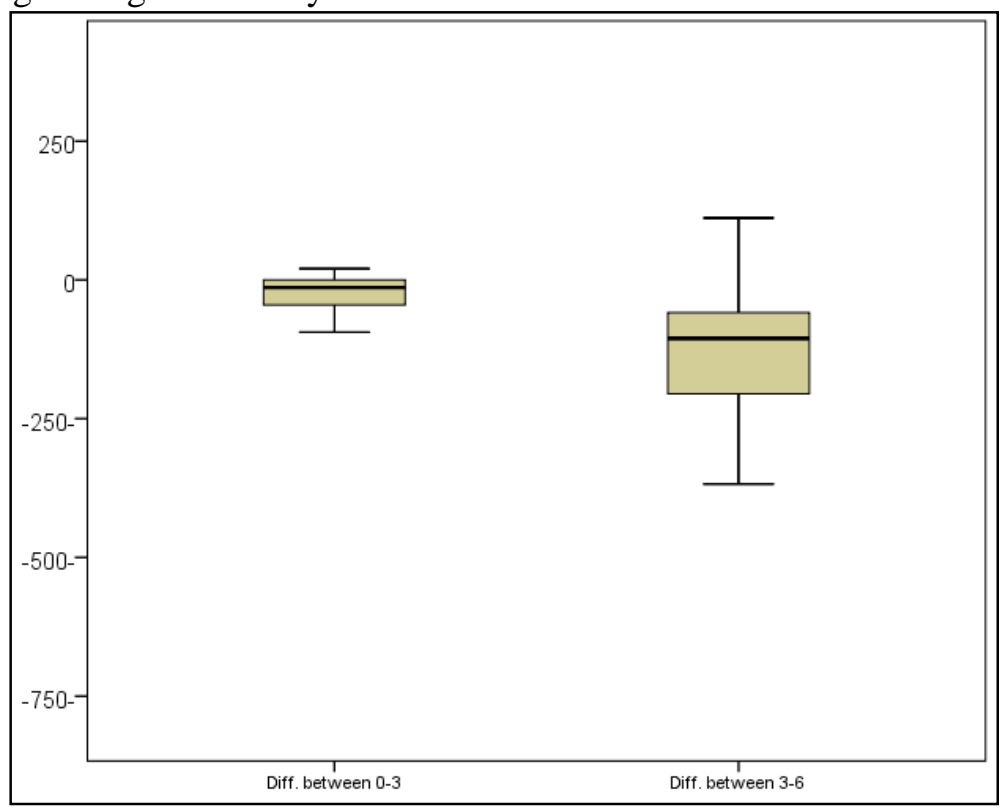

Figure (6): Comparison between the difference in serum FGF-23 level during the period of low-flux dialysis and the difference during the high-flux dialysis

Table (3): Comparison between the difference in echocardiography findings during the period of low-flux dialysis and the difference during the high-flux dialysis as regard the ejection fraction and right ventricular systolic pressure.

\begin{tabular}{|c|c|c|c|c|c|c|}
\hline & & $\begin{array}{c}\text { Diff. } \\
\text { between 0-3 }\end{array}$ & $\begin{array}{c}\text { Diff. } \\
\text { between 3-6 }\end{array}$ & $\begin{array}{c}\text { Test } \\
\text { value } \neq\end{array}$ & $\begin{array}{c}P- \\
\text { value }\end{array}$ & Sig. \\
\hline \multirow{2}{*}{$E F$} & Median (IQR) & $0(-3-1)$ & $1(0-2)$ & \multirow{2}{*}{-2.158} & \multirow{2}{*}{0.031} & \multirow{2}{*}{$S$} \\
\hline & Range & $-8-3$ & $-12-5$ & & & \\
\hline \multirow{2}{*}{$R V S P$} & Median (IQR) & $-1(-3-2)$ & $1(-3-3)$ & \multirow{2}{*}{-0.840} & \multirow{2}{*}{0.401} & \multirow{2}{*}{ NS } \\
\hline & Range & $-8-27$ & $-9-15$ & & & \\
\hline
\end{tabular}




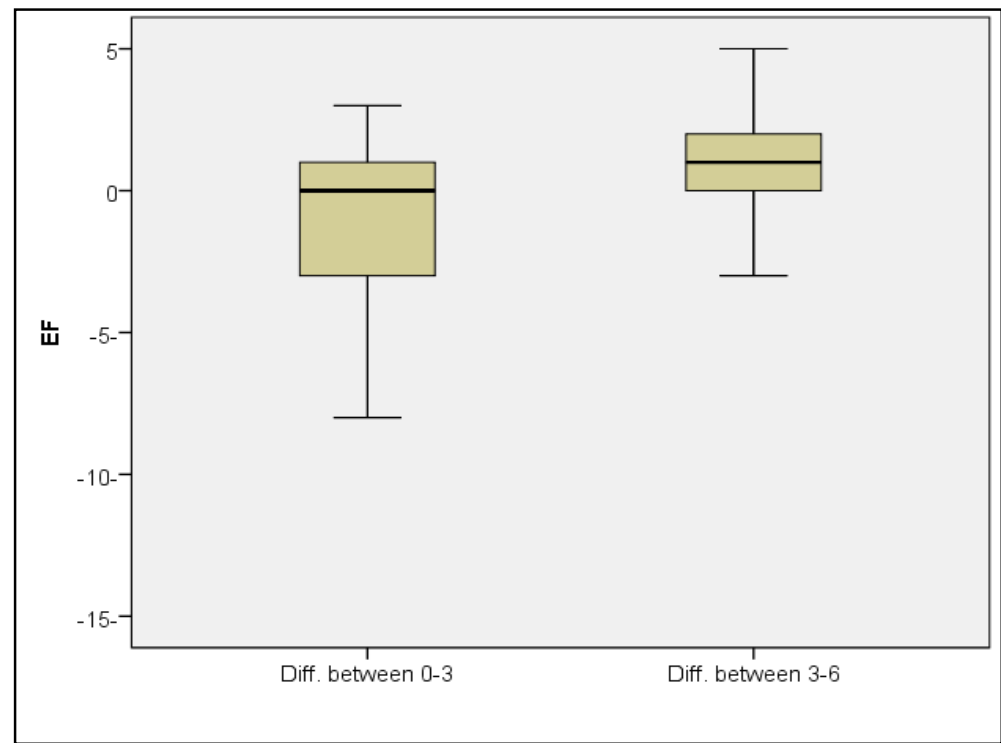

Figure (7): The difference in ejection fraction during the period of low-flux dialysis and the difference during the high-flux dialysis

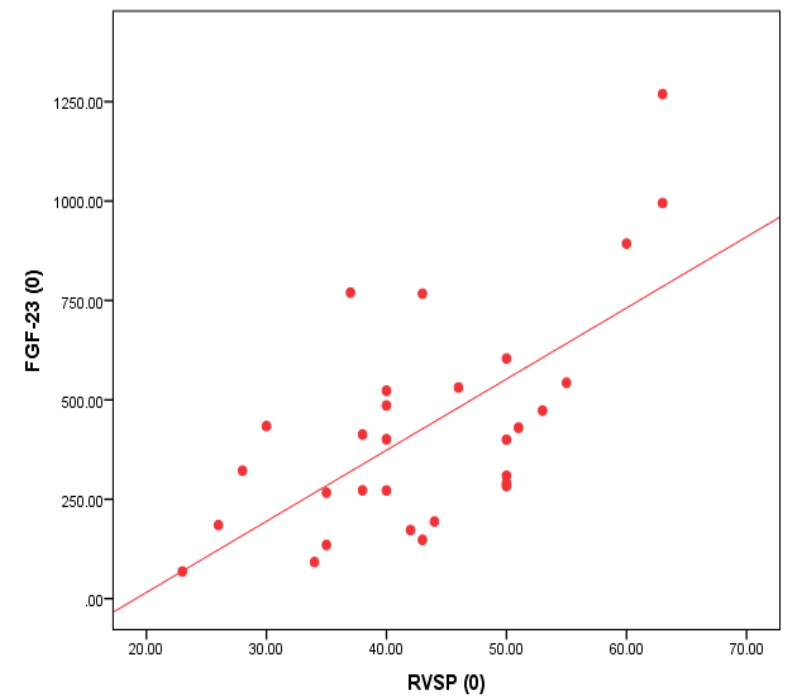

Figure (8): Correlation between FGF-23 and RVSP.

Table (4): Incidence of cardiac valve calcification in patients with higher FGF-23 levels:

\begin{tabular}{|c|c|c|c|c|c|c|}
\hline \multicolumn{2}{|c|}{ Baseline } & $\begin{array}{c}\text { Negative } \\
\text { Valvular } \\
\text { Calcification }\end{array}$ & $\begin{array}{c}\text { Valvular } \\
\text { Calcification }\end{array}$ & $\begin{array}{l}\text { Test } \\
\text { value }\end{array}$ & $\begin{array}{c}\text { P- } \\
\text { value }\end{array}$ & $\begin{array}{c}\text { Si } \\
\text { g. }\end{array}$ \\
\hline $\begin{array}{c}F G F-23 \\
(p g / m l)\end{array}$ & $\begin{array}{c}\text { Median } \\
\text { (IQR) }\end{array}$ & $283(185-413)$ & $543(429.9-893)$ & $-2.862 \ddagger$ & 0.004 & HS \\
\hline
\end{tabular}

Table (4) showed that the group of patients with cardiac valve calcification showed higher levels of serum FGF-23 (Figure 9). 


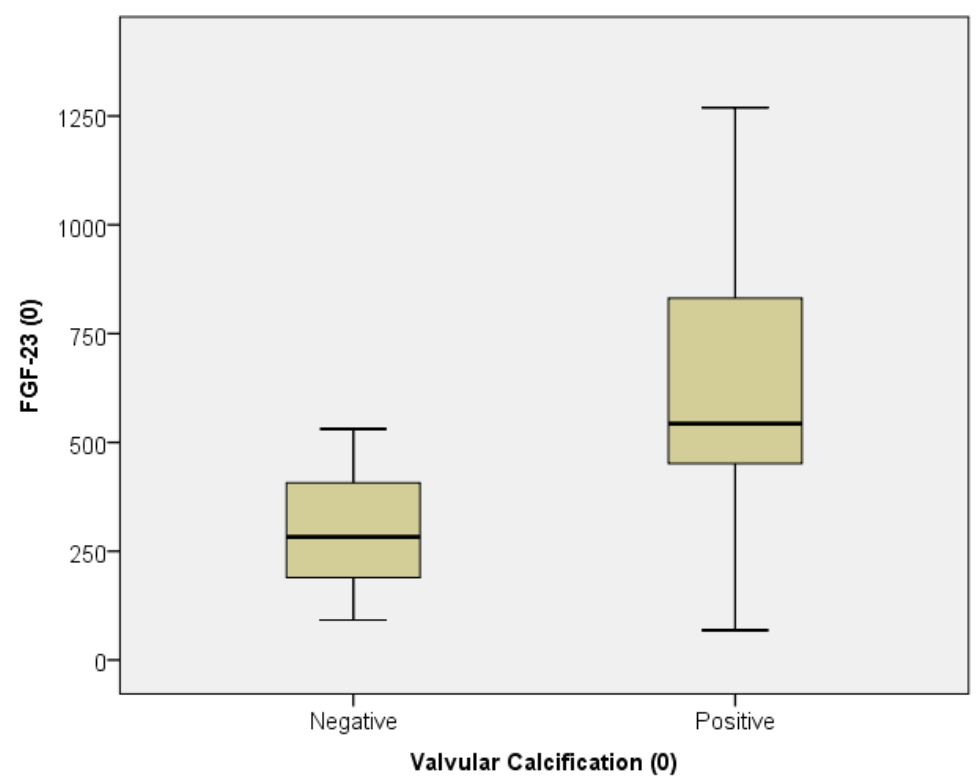

Figure (9): a Box plot showing a higher FGF-23 levels in patients with cardiac valve calcification. Table (5): Correlation coefficient (r) between FGF-23 and Lipid profile:

\begin{tabular}{|c|c|c|}
\hline \multicolumn{3}{|c|}{ Correlation } \\
\hline \multirow{2}{*}{ Baseline } & \multicolumn{2}{|c|}{ FGF-23 } \\
\hline & $\mathbf{R}$ & P-value \\
\hline $\begin{array}{c}\text { Total Cholesterol (mg/dl) } \\
\text { ( up to 200) }\end{array}$ & $-0.365^{*}$ & 0.048 \\
\hline $\begin{array}{c}\text { Triglyceride }(\mathrm{mg} / \mathrm{dl}) \\
(<150)\end{array}$ & -0.146 & 0.441 \\
\hline $\begin{array}{c}\boldsymbol{L D L}(\boldsymbol{m g} / \boldsymbol{d l}) \\
(<100)\end{array}$ & $-0.384 *$ & 0.036 \\
\hline $\begin{array}{c}\text { HDL (mg/dl) } \\
(40-60)\end{array}$ & -0.091 & 0.633 \\
\hline
\end{tabular}

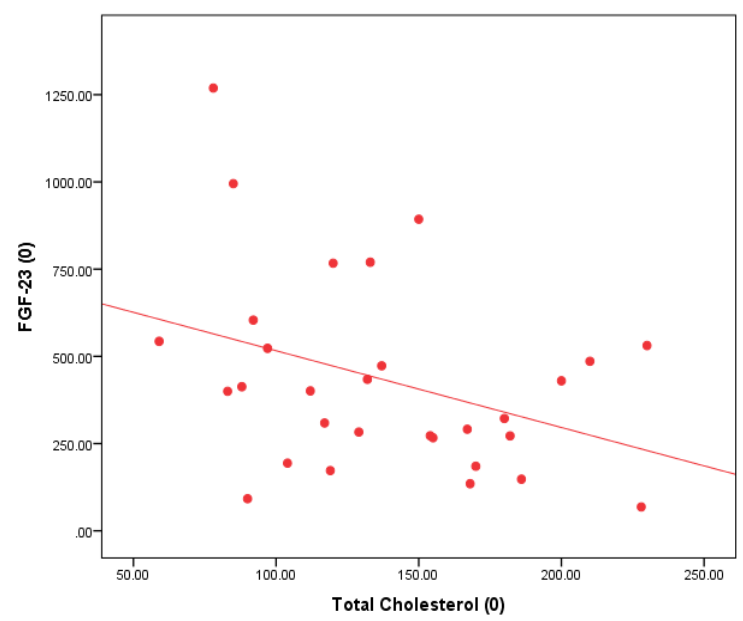

Figure (10): Correlation between FGF-23 and total cholesterol. 


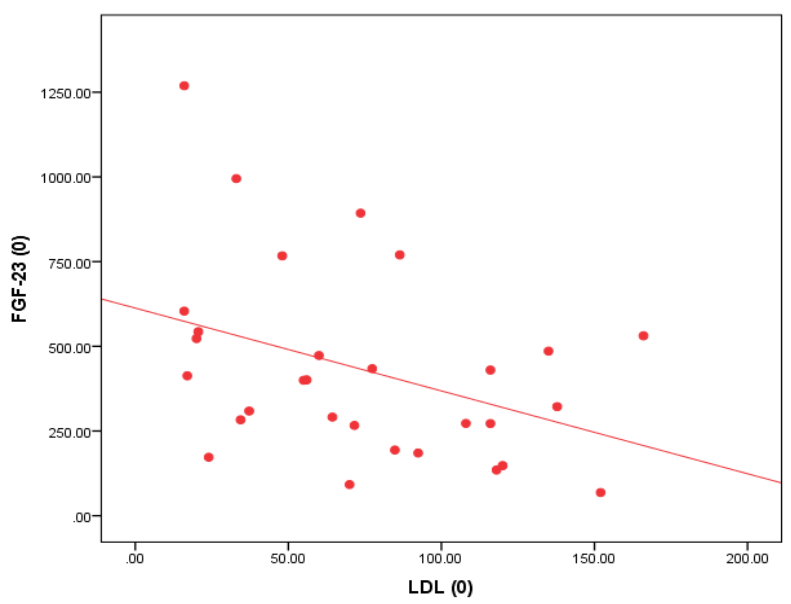

Figure (11): Correlation between FGF-23 and LDL.

\section{DISCUSSION}

Up to $40-50 \%$ of patients with end-stage renal disease (ESRD) die because of cardiovascular disease (CVD), reflecting their rapidly progressing atherosclerotic burden and heavy arterial calcification (6). Altered calcium, phosphorus, parathyroid hormone $(\mathrm{PTH})$, and vitamin D levels in ESRD induce mediators of bone-like metabolism and mineralization in the vascular tunica media ${ }^{(7)}$.

Fibroblast growth factor-23 (FGF-23) levels also start rising early in patients with chronic kidney disease and is implicated in cardiovascular and overall mortality of hemodialysis patients ${ }^{(\mathbf{8})}$. Elevated phosphate, calcium, PTH, and FGF23 levels are all associated with vascular calcification in patients with CKD and may directly promote calcification ${ }^{(\mathbf{9})}$.

Our study was conducted on 30 randomly selected prevalent hemodialysis patients aged $25-69$ years old with mean age of $50.10 \pm 11.54$ years. Male patients were $16(53.3 \%)$ and mean hemodialysis duration was $5.22 \pm 3.39$ years with hemodialysis duration ranging from 1 year to 13 years.

In our study, the baseline FGF-23 levels were higher than the normal range and this observation is in line with other reports of Gutierrez et al. ${ }^{(10)}$ and Jean et al. (11).

We found that there was a highly significant reduction of FGF-23 level with high-flux dialyzers than with low-flux dialyzers. The difference in the absolute change in FGF-23 levels between the three months period of low-flux dialysis and the three months period of high-flux dialysis showed a highly significant reduction with high-flux than with low-flux $(\mathrm{p}=0.000)$. This is in agreement with previous studies by $\mathbf{F u}$ et al. ${ }^{(12)}$ and Zhang et al. ${ }^{(13)}$.
There was no significant change in PTH during the three months of the low-flux period, the baseline and after three months PTH median was 330.85 with (IQR) $(163$ - 559.5) and 369.5 with (IQR) $(230-500)$ respectively $(\mathrm{p}=0.072)$. When using high-flux dialyzer, PTH levels showed a significant reduction from the start and after three months of the high-flux dialysis, from median (IQR) $369.5(230-500)$ to $274.9(183.8-482)(\mathrm{p}=0.021)$. The difference in the absolute change in PTH levels between the three months period of low-flux dialysis and the three months period of high-flux dialysis showed a significant reduction with high-flux than with low-flux ( $p=0.022)$. This is in agreement with Makar et al. ${ }^{(14)}$. This result was contradicting with the results of $\mathbf{F u} \boldsymbol{e t}$ al. (12) where their study showed no significant difference in calcium, phosphorus, $\mathrm{PTH}$, and alkaline phosphatase between low and high flux groups.

The difference in the absolute change in serum calcium level between the low-flux and the high-flux period showed significant reduction, $(\mathrm{p}=0.000)$. Also, the difference in the absolute change in serum phosphorus level between the low-flux and the highflux period showed significant reduction, $(p=0.006)$.

Hypoalbuminemia is common in patients with ESRD. It is caused by a combination of a reduced synthesis and an increased degradation of albumin. The altered albumin homeostasis in ESRD patients is caused by a systemic inflammatory state which correlates closely with mortality ${ }^{(\mathbf{1 5})}$. We found no significant change of serum albumin after the use of high-flux filters, as serum albumin levels changed from $3.85 \pm 0.55$ at start of high-flux period to $3.90 \pm$ 0.46 at the end of the 3-months high-flux period ( $\mathrm{p}=$ 0.351 ). When we compared the difference in the absolute change of serum albumin level during the 
low-flux period, which showed no significant change and the high-flux period (median -0.1, IQR (-0.2$0.1)$ ) and (median 0.05, IQR $(-0.1-0.3)$ ) respectively , it showed a significant increase in serum albumin when using high-flux compared to low-flux dialyzer ( $p=0.049$ ). The improvement in serum albumin with the use of high flux dialyzer could be due to a degree of enhanced serum albumin and nutritional state with high-flux dialyzers. This could be due to improved dietary intake as a result of potential removal of plasma substances that inhibit appetite. Leptin, which is one of middle-sized uremic toxins that weights $16000 \mathrm{D}$ could be removed with high flux dialyzer ${ }^{(16)}$. This is not in agreement with Makar et al. ${ }^{(14)}$ and El Arbagy et al. ${ }^{(17)}$.

As regards lipid profile, our patients had almost normal or high normal values of total cholesterol $(138.50 \pm 46.39 \mathrm{mg} / \mathrm{dl}$ ), normal LDL (median 70.8 (IQR $34.4-116 \mathrm{mg} / \mathrm{dl})$ ), elevated triglyceride (164.70 $\pm 62.65 \mathrm{mg} / \mathrm{dl})$ and decreased HDL (36.40 \pm 7.37 $\mathrm{mg} / \mathrm{dl})$. When using the difference in the absolute change between the low-flux and high-flux period there was a significant $(p=0.038)$ reduction of the total cholesterol during the high-flux period [median 8 (IQR $-5-31)$ ] and [median 4 (IQR -10 - 13)] respectively, but there was no significant difference in the absolute change of LDL, triglyceride, or HDL between lowflux and high-flux periods. This non-significant change of lipid profile in our study is contradicting with the findings observed by Wanner et $\boldsymbol{a l} .{ }^{(18)}$ who found that with high-flux polysulphone membranes, there was a higher reduction of triglyceride and total cholesterol than with high-flux modified cellulose membrane, while oxidized LDL decreased with highflux polysulphone but not with high-flux modified cellulose. This could be explained by the fact that polysulphones are more biocompatible than cellulose membranes causing less oxidative stress and not due to the flux permeability. Also, Schiffl \& Lang ${ }^{(19)}$ found that the improvement of uremic dyslipidemia is due to use of ultra-pure dialysate and not due to the flux permeability.

In our study, FGF-23 showed a negative correlation with baseline total cholesterol and LDL levels $(p=0.048)$ and $(p=0.036)$ respectively. This is in agreement with Yan et al. ${ }^{(20)}$. This correlation is thought to be due to the association of FGF23 with factors other than mineral metabolism as it belongs to the FGF19 subfamily and shares structural and biological similarities with the other family members (FGF19 and FGF21) that are involved in lipid and glucose metabolic homeostasis ${ }^{(21)}$.

In our study, there was no significant change in serum hemoglobin level or serum ferritin between the low-flux and the high-flux period $(\mathrm{p}=0.888)$ and $(\mathrm{p}=$ 0.322 ) respectively.

As regard, echocardiography findings, with the use of high-flux dialyzer there was a decrease in the number of patient with diastolic dysfunction even it did not reach a significant value. The adjusted difference between low-flux and high-flux periods showed a significance improvement $(\mathrm{p}=0.031)$ in ejection fraction value. This is in agreement with Francisco et al. (22). FGF-23 showed a positive correlation with RVSP at baseline and after 3 months on high-flux dialysis with the same P-value (0.000). There was a highly significant correlation $(\mathrm{p}=0.004)$ between FGF23 level and valvular calcification with low-flux dialyzer, and a significant correlation $(\mathrm{p}=$ 0.023 ) with the high-flux dialyzer. This is in agreement with Fu et al. ${ }^{(12)}$.

\section{CONCLUSION}

FGF-23 levels were significantly higher in hemodialysis patients than in normal population. FGF23 is better eliminated by high-flux dialyzers than lowflux dialyzers. FGF-23 is associated with increased incidence of cardiac valve calcification. FGF-23 positively correlates with right ventricular systolic pressure. There was a higher reduction of PTH, calcium, and phosphorus levels with high-flux dialyzers. There was a significant reduction of the total cholesterol with the high-flux dialyzer, but no significant change with LDL, HDL, or triglyceride was noticed.

\section{REFERENCES}

1. Jeppe HC, Erik BS, My S (2011): n-3 polyunsaturated fatty acids, lipids and lipoproteins in end-stage renal disease. Clinical Lipidology, 6 (5): 563-76.

2. Salusky IB, Goodman WG (2002): Cardiovascular calcification in end- stage renal disease. Nephrology Dialysis Transplantation, 17 (2): 336-9.

3. Liu S, Quarles LD (2007): How fibroblast growth factor 23 works. Journal of the American Society of Nephrology, 18 (6): 1637-47.

4. Gutiérrez OM, Mannstadt M, Isakova T et al. (2008): Fibroblast growth factor 23 and mortality among patients undergoing hemodialysis. New England Journal of Medicine, 359 (6): 584-92.

5. Wolf $\mathbf{M}$ (2012): Update on fibroblast growth factor 23 in chronic kidney disease. Kidney international, 82 (7): 73747.

6. Kraus MA, Kalra PA, Hunter J et al. (2015): The prevalence of vascular calcification in patients with endstage renal disease on hemodialysis: a cross-sectional observational study. Therapeutic advances in chronic disease, 6 (3): 84-96.

7. Moe SM, Chen NX (2008): Mechanisms of vascular calcification in chronic kidney disease. Journal of the American Society of Nephrology, 19 (2): 213-6. 
8. Anandh U, Mandavkar P, Das B, Rao S (2017): Fibroblast growth factor-23 levels in maintenance hemodialysis patients in India. Indian journal of nephrology, 27 (1): 9.

9. Adeney KL, Siscovick DS, Ix JH et al. (2009): Association of serum phosphate with vascular and valvular calcification in moderate CKD. Journal of the American Society of Nephrology, 20 (2): 381-7.

10. Gutiérrez OM, Mannstadt M, Isakova T et al. (2008): Fibroblast growth factor 23 and mortality among patients undergoing hemodialysis. New England Journal of Medicine, 359 (6): 584-92.

11. Jean G, Terrat JC, Vanel T et al. (2009): High levels of serum fibroblast growth factor (FGF)-23 are associated with increased mortality in long haemodialysis patients. Nephrology Dialysis Transplantation, 24 (9): 2792-6.

12. Fu X, Cui QQ, Ning JP, Fu SS et al. (2015): High-flux hemodialysis benefits hemodialysis patients by reducing serum FGF-23 levels and reducing vascular calcification. Medical science monitor: international medical journal of experimental and clinical research, 21:3467.

13. Zhang Z, Chen J, Sun $Z$ et al. (2015): effects of high-flux hemodialysis on FGF-23 and micro-inflammatory state in end-stage renal diseases patients. Zhonghua yi xue za zhi., 95 (26): 2074-8.

14. Makar SH, Sawires HK, Farid TM et al. (2010): Effect of high-flux versus low-flux dialysis membranes on parathyroid hormone. Iranian journal of kidney diseases, 4(4):327.
15. Haller $\mathbf{C}$ (2005): Hypoalbuminemia in renal failure: pathogenesis and therapeutic considerations. Kidney and Blood Pressure Research, 28 (5-6): 307-10.

16. Dhondt $A$, Vanholder $R$, Van Biesen W et al. (2000): The removal of uremic toxins. Kidney International, 58: S47-59.

17. El Arbagy AR, Koura MA, El AE et al. (2014): Study of effect of high-flux versus low-flux dialysis membranes on parathyroid hormone. American Journal of Clinical Medicine Research, 2 (1): 36-42.

18. Wanner C, Bahner U, Mattern R et al. (2004): Effect of dialysis flux and membrane material on dyslipidaemia and inflammation in haemodialysis patients. Nephrology Dialysis Transplantation, 19 (10): 2570-5.

19. Schiffl H, Lang SM (2010): Effects of dialysis purity on uremic dyslipidemia. Therapeutic Apheresis and Dialysis, 14 (1): 5-11.

20. Yan J, Zhang M, Ni Z et al. (2017): Associations of serum fibroblast growth factor 23 with dyslipidemia and carotid atherosclerosis in chronic kidney disease stages 3-5D. International Journal Of Clinical And Experimental Medicine, 10 (9): 13588-97.

21. Fukumoto $\mathbf{S}$ (2008): Actions and mode of actions of FGF19 subfamily members. Endocrine journal, 55 (1): 23 31.

22. Francisco RC, Aloha M, Ramón PS (2013): Effects of high-efficiency postdilution online hemodiafiltration and high-flux hemodialysis on serum phosphorus and cardiac structure and function in patients with end-stage renal disease. International urology and nephrology, 45 (5): 13738 . 